DOI: $10.24850 /$ j-tyca-2020-06-06

Artículos

\title{
Propuesta de metamodelo para la regulación de la demanda urbana de agua potable
}

\section{Proposed meta-model for the regulation of the urban demand for drinking water}

José Andelfo Lizcano-Caro ${ }^{1}$, https://orcid.org/0000-0003-1537-530X

Rubén Medina-Daza², https://orcid.org/0000-0002-9851-9761

Mario Guadalupe González-Pérez³, https://orcid.org/0000-0002-85110465

1Universidad Distrital Francisco José de Caldas, Bogotá, Colombia, jalizcanoc@udistrital.edu.co

2Universidad Distrital Francisco José de Caldas, Bogotá, Colombia, rmedina@udistrital.edu.co

3Universidad de Guadalajara, Guadalajara, México, inge_united@hotmail.com

Autor para correspondencia: Mario Guadalupe González Pérez, inge_united@hotmail.com

\section{Resumen}


En el mundo, la regulación para la prestación del servicio del acueducto se basa en los costos que requiera la empresa para llevarla a cabo, sobre todo en la demanda futura; ejemplo de ello es el indicador para calcular insumos y costos de producción del bien y servicio. En este sentido, la eficiencia, suficiencia financiera y presupuestal dependen en gran medida de la exactitud de las mediciones y proyecciones determinadas para un entorno de mercado. Dentro del proyecto de investigación doctoral "Modelo de regulación para agua potable en horizontes de largo plazo", se propuso un metamodelo para la regulación de la demanda urbana de agua potable en escenarios de equilibrio inestable, con criterios de solidaridad y priorización de inversiones ecoambientales. Metodológicamente, se aplicaron varias herramientas estadísticas para determinar cuál sería el mejor método. Por ello, se relacionó el cálculo de la demanda de agua y se encontró que deben manejarse determinantes, variables y otros criterios condicionados según la estructura del territorio y diferentes parámetros socioeconómicos. Además, el sistema de soporte de decisiones, auxiliado por un análisis de redes neuronales, se acomodó mejor a las condiciones de los complejos urbanos. Sin embargo, se tuvieron dificultades en la búsqueda de la información, ya que los metamodelos son una herramienta relativamente reciente en comparación con el registro de información en bases de datos del servicio de acueducto.

Palabras clave: metamodelo, agua potable, sistema de regulación.

\section{Abstract}


Globally, the regulation for the provision of the aqueduct service is based on the costs of providing the service of the company, especially in the future demand, an example of this is the indicator to calculate inputs and costs of production of the good and service. In this sense, efficiency, financial and budgetary sufficiency depends to a large extent on the accuracy of measurements and projections determined for a market environment. Within the doctoral research project "Regulatory Model for drinking water in long-term horizons", it was proposed a meta-model of urban demand for drinking water in scenarios of unstable equilibrium, with solidarity criteria and prioritization of eco-environmental investments. Methodologically, several statistical tools were applied to determine the best method. Therefore, the calculation of water demand was related and it was found that the determinants, variables and other criteria conditioned by the structure of the territory and the different socioeconomic parameters should be managed. In addition, the decision support system aided by a neural network analysis, was better suited to the conditions of urban complexes. However, there were difficulties in the search for information, since the meta-models are a relatively recent tool in comparison to the registration of information in aqueduct service databases.

Keywords: Meta-model, drinking water, regulation system.

Recibido: 03/04/2019

Aceptado: 23/03/2020 


\section{Introducción}

En la era moderna, las principales innovaciones se han dado en el servicio de alcantarillado, pretendiendo alcanzar la misma cobertura que el sistema de acueducto, enfocando sus intereses en el aspecto sanitario, aunque en el servicio de acueducto, los principales avances han ocurrido en el mejoramiento de procesos de tratamiento de agua. En este sentido, un tema de importancia urbana es la proyección de la demanda de agua potable y las diferencias entre sus tipos, como es el caso de las demandas de agua potencial y la efectiva. Para abordar esta temática, se revisaron reglamentos técnicos del sector de agua potable y saneamiento básico (Ministerio de Ambiente, Vivienda y Desarrollo Territorial, 2003), en el que se resalta el cálculo y las variables de la demanda para obtener la demanda total, la cual se obtiene mediante la determinación de la demanda media residencial y la demanda media de otros usos, teniendo en cuenta aspectos como la demanda máxima horaria general y la demanda máxima diaria de la población de estudio. También se consultaron temáticas para la aplicación del metamodelo, como las variables a utilizar, la identificación de los distintos modelos que existen para su cálculo, para así determinar cuál es el más adecuado. 


\section{Aspectos económicos relacionados con el recurso hídrico}

El primer acercamiento de la economía en los recursos naturales y del recurso hídrico se encuentra en el concepto de "la economía ambiental", que a partir del año 1970 comenzó a tratar temas como la sostenibilidad y la economía para entornos ambientales; pero según algunas referencias (Pearce \& Turner, 1990), desde el año 1990 se trata el tema alrededor de la política monetaria del medio ambiente y sus implicaciones en el mercado. Nijkamp (1977) define a esta economía como el estudio científico de los aspectos relacionados con la escasez y el comportamiento humano, en relación con su entorno natural, físico y residencial. Una definición más reciente es de Gilpin (2000), quien estudió la economía ambiental y sus implicaciones en todos los costos inherentes al deterioro y control del ambiente, aparte de la totalidad de los beneficios derivados de la protección de los recursos y el ambiente en un esquema global de costo-beneficio, con equilibrio de éstos en cada sector, fortaleciendo, de una o de otra manera, la base de recursos a la que recurrirán las generaciones presentes y futuras. Surge así la necesidad en equiparar a la llamada nueva economía del agua con la privatización de la producción, distribución y gestión de los recursos hídricos. De esta manera, se podría clasificar el recurso hídrico según sus fuentes de abastecimiento, administración estatal y municipal, por los usos que se le proporcionan. 


\section{Usos del agua}

Por largo tiempo han prevalecido otros elementos que implicaron cambios, por esta razón aumentó el uso de las aguas por múltiples variables, y por el incremento de las necesidades. Por ende, los usos y la demanda también cambiaron. Entre los sectores destaca el industrial, los servicios (bebidas, saneamiento, limpieza, recreación) y las actividades agrícolas.

\section{Recursos hídricos}

Actualmente, en muchas áreas del planeta no se cumplen las pautas sostenibles para el uso y usufructo del recurso hídrico. La creciente demanda del recurso, al igual que la reducción de los caudales en ríos traen graves consecuencias para usuarios y ecosistemas; la sobreexplotación de acuíferos a tasas superiores a la reposición natural; los problemas de contaminación y degradación de la calidad de las aguas, y las dificultades de acceso al recurso para satisfacer necesidades básicas de un alto porcentaje de la población son desafíos que exigen con urgencia 
estrategias que permitan resolver las numerosas tareas pendientes en cuanto al uso de los recursos hídricos. En la actualidad se puede conocer la capacidad máxima de oferta hídrica que es posible brindar para satisfacer las necesidades en el ámbito nacional.

La oferta del recurso hídrico disponible ha disminuido de manera significativa en razón del aumento de la demanda de la población mundial (OMS, 2015). En 2015 existían alrededor de 663 millones de personas que no contaban con acceso a agua potable, 1800 millones en todo el mundo utilizaban una fuente que estaba contaminada.

La escasez en el orbe alcanzó niveles críticos hacia 2015, a causa de fenómenos meteorológicos atribuidos al cambio climático y al crecimiento incesante de la población. La correlación demanda-oferta de agua en Colombia muestra el uso doméstico o humano con un $17.8 \%$ (184 $253865 \mathrm{~m}^{3}$ ). Por ello, la proyección de la demanda a largo plazo es una herramienta que ayuda a identificar el volumen de agua necesario para satisfacer las necesidades y así mejorar la cobertura en áreas urbanas, con la planeación adecuada del ordenamiento territorial. Del mismo modo, permite tomar acciones correctivas que disminuyan la demanda debido a su constante e incesante aumento.

\section{Proyección de la demanda de agua potable}


La investigación acerca de la proyección de la demanda en los servicios públicos presenta oportunidades en el campo de la ingeniería, tecnología y de los procesos medioambientales, por ejemplo, el tiempo que requiere operar una planta de tratamiento de agua potable, así como proveer nuevas fuentes de este importante líquido cuando la demanda crezca a largo, mediano o corto plazos. Así, los índices de escasez del agua pueden determinarse y se conoce el ingreso proveniente de partir de dicho índice (Cutore, Campisano, Kapelan, \& Savic, 2007).

La hipótesis planteada en el documento supone un metamodelo de variación de regresión lineal básica, que emplea una interfaz de interpretación para registros de demanda de agua, así como sus determinantes en la proyección de agua potable en horizontes a largo plazo para entornos urbanos; esto, como una herramienta que aumenta la calidad y cantidad de posibilidades de regulación en el sector de la prestación de agua potable, y en la eficiencia financiera, operativa y administrativa de las empresas que prestan el servicio de acueducto.

El régimen de libertad regulada requiere de proyecciones futuras de demanda de agua potable. Con esta función y las pertinentes a la suficiencia y eficiencia financiera, es posible modelar costos medios de largo plazo, incluyendo los incrementales, causados por demandas marginales. Para responder a estas exigencias y a las distorsiones climáticas, ambientales y socioeconómicas, se construyó el modelo aquí expuesto, con base en escenarios de equilibrio inestable que consideraba criterios de solidaridad y de priorización de inversiones ecoambientales. Para ello, se plantearon dos posibles herramientas de pronóstico entre el periodo 2006-2040, para el caso particular de la ciudad de Bogotá, 
Colombia: 1) redes neuronales, y 2) series de tiempo, de las que sólo esta última generó resultados validables; se encontró que los estratos 1 y 2 muestran incrementos en la demanda, mientras que los estratos 3, 4, 5, 6 y el sector comercial tienden a decrecer en su consumo.

Los principales modelos de pronóstico investigados fueron los siguientes: modelo de serie temporal, utilizado para predecir el futuro con pleno conocimiento de los acontecimientos recientes a través de las estadísticas; modelo econométrico, para predecir variables relacionadas con la economía como demanda, oferta y precio, así como su predicción de prejuicios, la cual se efectuó debido a la falta de hechos históricos o cuando un nuevo producto o competidor ingresa al mercado (Hamilton, 1994).

El modelo de proyección de demanda de agua planteado incluyó dos estructuras: fija y variable. La estructura fija presenta tres componentes particulares, que son capacidad administrativa, gerencial y la planta física. La estructura variable, por su parte, presenta cuatro componentes: elementos financieros, emergentes, operativos, y meteorológicos y/o climáticos. El modelo contiene elementos esenciales de diseño, que son Add on (o entradas), buscador, editory gestor de restricciones.

A través del modelo se buscó realizaruna proyección de la demanda de agua para consumo humano, con el fin de determinar el efecto que tienen las determinantes identificadas con base en la revisión de estudios en el mundo. En este aspecto se concluyó que para Colombia, las determinantes a considerar son las siguientes: a) el clima, con sus variables de temperatura y precipitación; b) la estructura tarifaria, con su variable "precio regulado del agua"; c) el cambio climático, medido por 
los fenómenos El Niño y La Niña, y d) las características de la vivienda con la variable densidad, referente al número de personas por vivienda. Conforme al procesamiento de estas determinantes, se buscó obteneruna medida del efecto que tienen en la proyección de la demanda de agua para Colombia, en horizontes de largo plazo. El tratamiento de variables explicativas y explicadas se indica en la Tabla 1.

Tabla 1. Variables explicativas y explicadas.

\begin{tabular}{|c|c|c|c|c|}
\hline & Variable & Descripción & Unidades & Fuente \\
\hline \multirow[t]{5}{*}{ Explicativas } & Añ̃o & $2006-2016$ & & Acueducto \\
\hline & Mes & $\begin{array}{c}\text { Enero- } \\
\text { diciembre }\end{array}$ & & Acueducto \\
\hline & Estrato & $\begin{array}{c}1-6, \\
\text { comercial, } \\
\text { oficial, } \\
\text { industrial, } \\
\text { especial }\end{array}$ & & Acueducto \\
\hline & $\begin{array}{c}\text { Valor } \\
\text { facturado }\end{array}$ & $\begin{array}{c}\text { Valor } \\
\text { facturado por } \\
\text { unidad de } \\
\text { consumo }\end{array}$ & $\begin{array}{c}\text { COP } \\
\text { (pesos } \\
\text { Colombia- } \\
\text { nos) }\end{array}$ & Acueducto \\
\hline & Temperatura & $\begin{array}{l}\text { Medianas de } \\
20 \\
\text { estaciones }\end{array}$ & ${ }^{\circ} \mathrm{C}$ & IDEAM \\
\hline
\end{tabular}




\begin{tabular}{|c|c|c|c|c|}
\hline & Precipitación & $\begin{array}{c}\text { Medianas de } \\
34 \\
\text { estaciones }\end{array}$ & $\mathrm{Mm}$ & IDEAM \\
\hline & Población & $\begin{array}{c}\text { Proyecciones } \\
\text { de población } \\
1985-2020\end{array}$ & Personas & DANE \\
\hline Explicada & $\begin{array}{l}\text { Consumo } \\
\text { promedio }\end{array}$ & $\begin{array}{c}\text { Consumo } \\
\text { promedio de } \\
\text { agua }\end{array}$ & $\mathrm{m}^{3} / \mathrm{s}$ & Acueducto \\
\hline
\end{tabular}

Para las variables de temperatura y precipitación, se tomó la mediana de las estaciones, pues estas medidas tienen muchos valores atípicos.

Se creó una variable de intervención "I_ESPECIAL", para modelar el cambio antes y después de 2010, que sufrió el consumo de agua en el estrato ESPECIAL; ésta es una variable dicotómica que toma el valor 0 antes de 2010, y 1 después de ese año. Se ajustó un modelo de regresión para las proyecciones de población del DANE y se contempló de manera mensual.

\section{Materiales y métodos}


En los diferentes modelos investigados acerca del cálculo del suministro de agua se muestra que el horizonte de proyección tiende a mantener equilibrada la demanda de agua en el corto y largo plazos. Sin embargo, existe una dependencia de algunas variables y determinantes que se utilizan, así como de las diferentes condiciones en la ubicación de la zona donde se realizan los cálculos. Cabe señalar que no se les dio prioridad dentro del análisis a los modelos para proyección de agua potable a corto plazo, pues al incluir variables que afectan la demanda diaria, las proyecciones pierden su alcance y capacidad de decisión.

Dentro de la literatura consultada (31 casos analizados), se enlistan a continuación los modelos encontrados, junto con su frecuencia en orden de uso, de mayor a menor:
a) Análisis de regresión (25\%).
b) Análisis de redes neuronales (25\%).
c) Sistema auxiliar de toma de decisiones (16\%).
d) Sistemas basados en escenarios (6\%).
e) Modelos econométricos (6\%).
f) Modelo entrópico bayesiano (3\%).

De los dos documentos consultados para el caso colombiano, el primero se emplea como modelo de regresión y el segundo como un método econométrico de sección transversal. Entre los múltiples 
documentos revisados, evaluados y criticados están los de "IDEAM (2010), Índice de Alteración Potencial de la Calidad de Agua en condiciones hidrológicas de año medio, subzonas hidrográficas. Bogotá: IDEAM" e "IDEAM (2015)), Estudio Nacional de Agua 2014, Bogotá". Estas investigaciones proporcionaron información para establecer proyecciones de niveles de riesgo en suministro de agua y para la construcción de escenarios de cambio climático, para fenómenos de La Niña y El Niño.

Igualmente, se tuvo acceso a las bases y constructos de los estudios que soportaron la regulación de agua potable en Colombia, expedida s por la Comisión de Regulación de Agua Potable y Saneamiento Básico-CRA, para los tres periodos tarifarios regulados desde 1994: primer periodo, Resolución 08 de 1995; segundo periodo, Resolución 287 de 2004, y tercer periodo, Resolución 688 de 2014. Uno de estos estudios fue el siguiente: "Comisión de Regulación de Agua Potable y Saneamiento Básico (2014). Marco tarifario para los servicios públicos de Acueducto y Alcantarillado. Estándares de Servicio y Eficiencia. Bogotá: CRA". Otro de los estudios de soporte fue "Investigación al régimen tarifario de agua potable y saneamiento básico. Bogotá, D.C." (Lizcano, 2011). Este libro contiene análisis de series y construcción de datos desde 1994 hasta 2010. Tal investigación duró siete años y expone una de las bases para el sustento científico de este artículo.

\section{Planteamiento del metamodelo}


Con base en el esquema de la demanda requerida se planteó uno nuevo, que incluyera herramientas para generar un modelo y después el metamodelo. El esquema propone la generalidad de la demanda a partir del consumo del usuario como sujeto base para la estimación del mismo y como base fundamental el caudal, siguiendo un esquema de cadena de suministro (o número de la referencia), el cual es el proveedor del ins umo para la prestación del servicio.

El siguiente proceso se muestra en la Figura 1, seguido de un planteamiento del modelo, donde se asignaron funciones a cada una de las determinantes. Durante la fase de entrenamiento se buscó estandarizar los resultados y, por último, establecer unos límites de aplicación de la regla. 


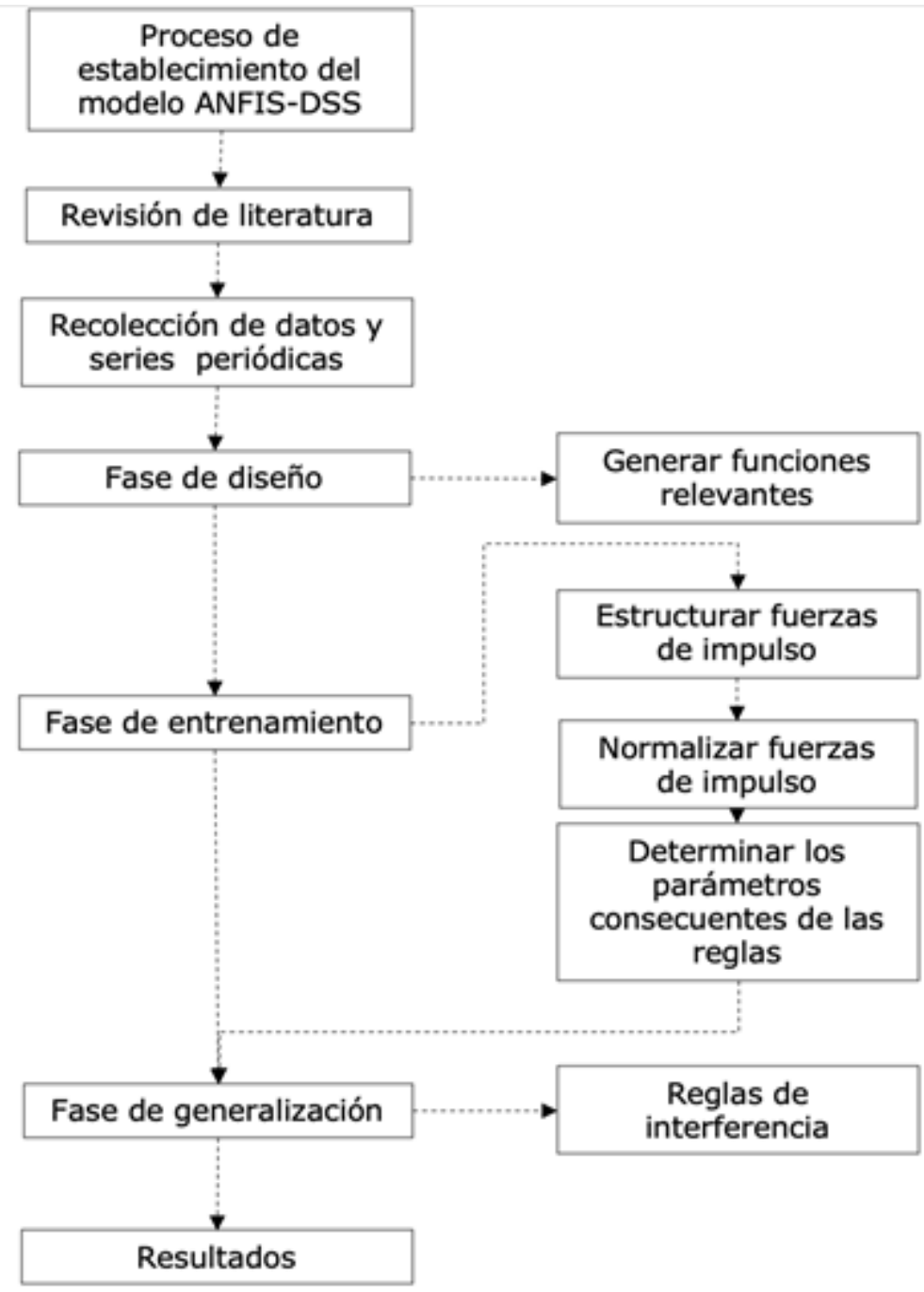

Figura 1. Esquema de referencia para planteamiento de metamodelo. Fuente: elaboración propia (2019).

Las determinantes del modelo seleccionadas en los ámbitos nacional e internacional relacionan el cálculo de la demanda de agua. Se encontró que deben manejar determinantes, variables y otros criterios. Estos componentes deben estar condicionados según la estructura del territorio 
y los diferentes parámetros socioeconómicos. Por medio de las distintas consultas se considera que, para países como Colombia, aplican los siguientes criterios: clima, temperatura y precipitación, entre otros, los cuales se explican como parte de los resultados del planteamiento.

Partiendo de los preceptos y precedentes establecidos es necesario resaltar que el hecho de que se utilice un método u otro no implica que sea más o menos efectivo, sino que se ajusta mejor a condiciones específicas; por tanto, con base en la posibilidad de optimizar modelos de regresión a través de redes neuronales, que en general son capaces de almacenar conocimiento a partir de la experiencia e integrando dicho modelo en un sistema auxiliar de toma de decisiones, se aproxima a lo que se busca con el método de proyección de demanda.

\section{Resultados}

Para la aplicación de las redes neuronales se entiende que existen unidades únicas llamadas neuronas, que tienen un mecanismo de funcionamiento, el cual se explica a continuación:

$K$ hace referencia a la neurona, la cual procesa una entrada $x$, a través de un activador de función $\varphi$, generando una salida $w$. Estableciendo dos relaciones con o sin límites: 
$\mathrm{u}_{\mathrm{k}}=\sum_{\mathrm{j}=1}^{\mathrm{p}} \mathrm{w}_{\mathrm{kj}} \mathrm{x}_{\mathrm{j}}$

$\mathrm{y}_{\mathrm{k}}=\varphi\left(\mathrm{u}_{\mathrm{k}}-\theta_{\mathrm{k}}\right)$

Donde la Ecuación (1) y la Ecuación (2) representan la función general de la neurona con una entrada y una salida:

$U_{k}$ : salida con comportamiento lineal de información de la neurona.

$W_{k j}$ : salida que arroja la neurona producto de un procesamiento.

$X_{j}$ : entrada de información que se le introduce a cada neurona.

La Ecuación (2) usa la salida lineal para obtener un comportamiento similar al esperado, donde:

$U_{k}$ : salida con comportamiento lineal de información de la neurona. $\theta_{k}$ : función de límite de la salida.

$\varphi$ : función de activación.

$y_{k}$ : señal de la salida lineal de la neurona.

Cada neurona posee conectividad con sus semejantes, a través de coeficientes de variación. La fortaleza de su aprendizaje depende de su capacidad de agruparse, por ello se disponen en capas. Hay capas con 
funciones específicas, como las de entrada y las de salida. La capa de entrada consiste en neuronas que reciben información del entorno externo. La capa de salida consiste en neuronas que comunican la salida del sistema al usuario o externa al entorno. Inclusive existen capas intermedias, con distintas funciones de procesamiento, por ejemplo, cuando la relación entre los registros de entrada no es lineal, las capas intermedias buscan generalizar características, de modo que la información de salida lo sea (Figura 2 y Figura 3).

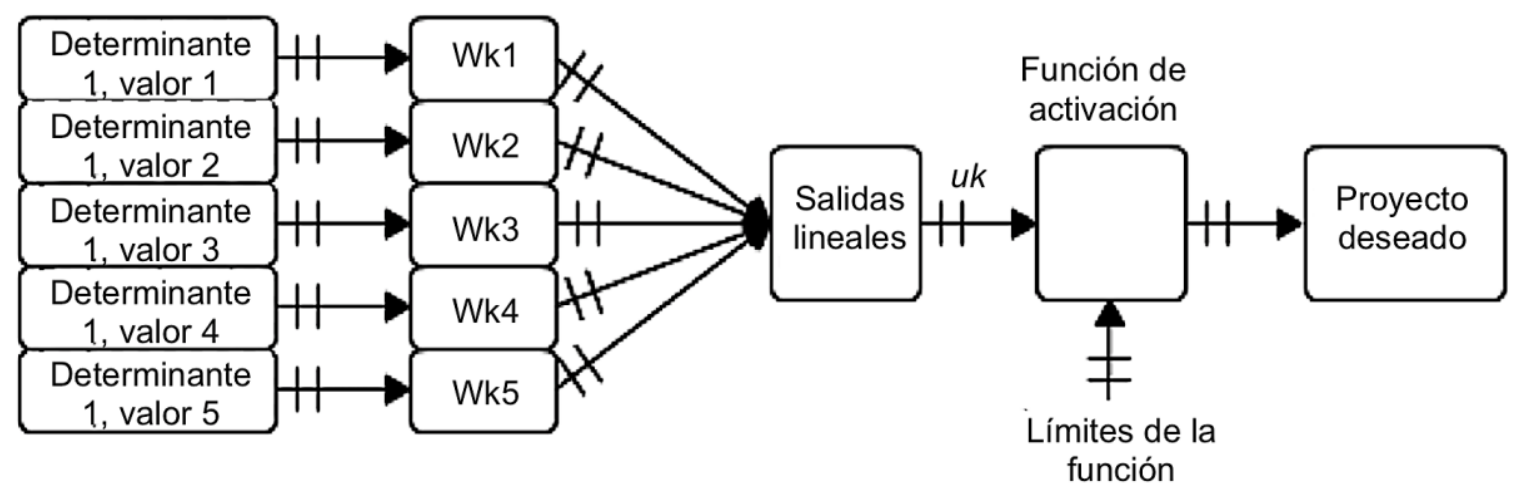

Figura 2. Estructura de la red neural. Fuente: Kuo and Xue (1998). 
Tecnología y

Ciencias $₫$ Agua
2020, Instituto Mexicano de Tecnología del Agua

Open Access bajo la licencia CC BY-NC-SA 4.0

(https://creativecommons.org/licenses/by-nc-sa/4.0/)

\section{Proyección deseada}

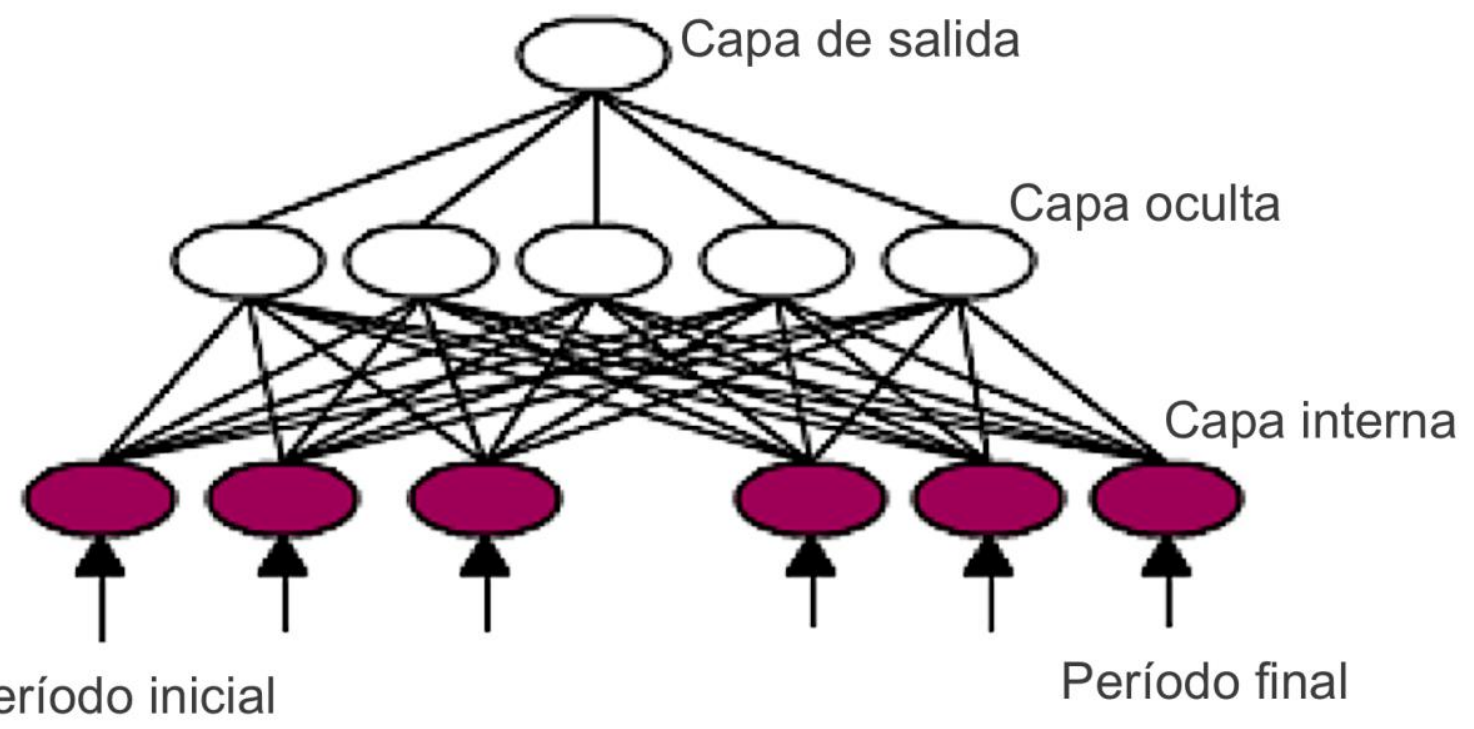

Figura 3. Estructura de la red neu al por capas. Fuente: Tomado y modificado de Kuo y Xue (1998).

\section{Cálculo de errores}

El error del método se produce entre las conexiones estratificadas de la red neural, que debe ser calculado y minimizado. Por otro lado, el proceso de aprendizaje se lleva a cabo de dos maneras: la primera, supervisada o controlada (como la regresión lineal); mientras que la segunda es autónoma. El proceso de minimización puede variar gracias a la 
versatilidad que ofrece cada uno, para ello se consideran métodos como los de gradiente descendente, gradiente conjugado, Quasi-Newton, y métodos de Levenberg-Marquardt, que en general se caracterizan por ser iterativos, con cálculos de mínimos. Uno de los temas críticos está en la determinación de capas internas y su composición, donde Lenard, Alam y Madey (1995) proponen una generalidad, sobre la cual se acepta que el número de capas ocultas debe estar dado por el patrón $2 n+1$, donde $n$ es el número nodal. Otros autores (Patuwo, Hu, \& Hung, 1993) proponen que sea el $75 \%$ de la cantidad de nodos totales. La introducción de una función que proporcione un comportamiento no lineal a los datos ayuda a dar una proyección más real que ideal. Esto se puede usar mediante un proceso de retropropagación, donde a través de una fase supervisada se introduce un patrón a la capa neuronal de salida, que en una segunda fase se replica en fase reversa hacia las capas internas, promoviendo la generalización de patrones a nivel externo:

$\mathrm{n}=\sum_{\mathrm{i}=1}^{\mathrm{i}=\mathrm{q}}\left(\mathrm{w}_{\mathrm{i}} * \mathrm{p}_{\mathrm{i}}\right)+\mathrm{b}$

En la fórmula anterior (Ecuación (3)) se realizó la sumatoria de una función lineal que simula una función de imitación, donde wi representa el peso del vector; $p_{i}$, el vector de entrada, y $b$ es un factor de corrección o ajuste (Figura 4). Posteriormente se aplica una función de transferencia para las demás capas (Michie, Spiegelhalter, \& Taylor, 1994). 
Tecnología y

Ciencias $₫$ Agua
2020, Instituto Mexicano de Tecnología del Agua

Open Access bajo la licencia CCBY-NC-SA 4.0

(https://creativecommons.org/licenses/by-nc-sa/4.0/)

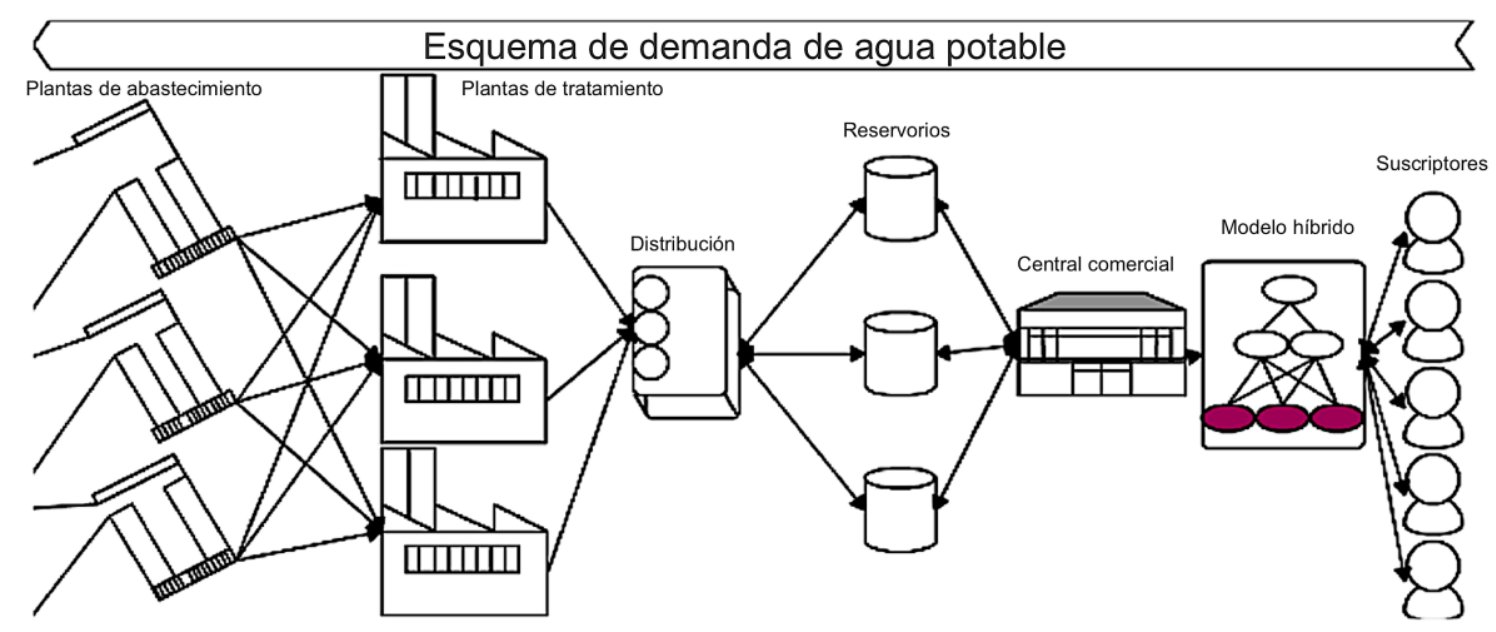

Figura 4. Esquema de demanda de agua potable en niveles de demanda.

Su efectividad depende de tres coeficientes:

1. Coeficiente de tasa de aprendizaje: regula la velocidad con la que se pueden cambiar los pesos a través del tiempo, reduciendo la posibilidad de cualquier oscilación de peso durante el ciclo de entrenamiento de las neuronas y de las capas.

2. Condiciones de momento o impulso: controla cuántas iteraciones resiste la corrección de un error, para determinar así su capacidad de impulso y medir su alcance.

3. Condiciones de salida o finalización de ciclos de entrenamiento: pueden asignarse a través de métodos de fijación de tiempos límites, personalizar el número máximo de ciclos de aprendizaje o usar precisión mínima. 


\section{Planteamiento del modelo}

Las determinantes aplicables para el modelo:

\section{Clima}

Está asociado con la temperatura y precipitación. Antes de 1985, el clima se consideraba una variable independiente; sin embargo, a partir de diversos estudios surgió la necesidad de modelarla como se hizo en 2005 mediante el método ARIMA (Autoregressive Integrated Moving Average), que toma variables enfocadas al clima. Este método permitió realizar mejores aproximaciones que las que se hacían con regresiones lineales o métodos univariados (Bougadis, Adamowski, \& Diduch, 2005; Adamowski, 2008; Caiado, 2010). Dependiendo de las condiciones del territorio, pueden variar de acuerdo con la región y ubicación geográfica; dicha variabilidad tiene implicaciones sobre la demanda. Esta determinante para su aplicación tendrá dos variables: temperatura y precipitación. 


\section{Temperatura}

Con base en estudios de caso, como el de Phoenix (Balling \& Gober, 2007), se relaciona un aumento de $1^{\circ} \mathrm{C}$ en la temperatura anual al aumento de $6.6 \%$ en la demanda anual de agua per cápita. Esto quiere decir que la temperatura tiene influencia en la demanda de agua, ya que con la variabilidad del mismo, y teniendo en cuenta las distintas zonas del país, este factorcambiaría, siendo relevante para el cálculo de la demanda de agua. Por ejemplo, para la ciudad de Manizales, durante el primer método, la demanda de agua mensual disminuye $2 \%$ por cada incremento de $1^{\circ} \mathrm{C}$ en la temperatura; para el segundo método, la demanda de agua mensual disminuye en $0.23 \%$ por cada incremento de $1^{\circ} \mathrm{C}$ en la temperatura.

\section{Precipitación}

Según los estudios de casos investigados, como el de Phoenix (Balling, \& Gober, 2007), se explica la disminución de $10 \%$ en la precipitación resultante de un aumento de $3.9 \%$ en la demanda anual de agua per cápita. Estas cifras son relevantes, pues en Colombia existe una 
variabilidad que depende de las zonas del país, como se muestra en la Figura 5. En el caso de la ciudad de Manizales, y de acuerdo con los modelos utilizados para el primer método, la demanda de agua mensual disminuye de $0.03 \%$ por un incremento de $1 \mathrm{~mm}$ en la precipitación, y para el segundo método baja en $0.001 \%$, por un incremento de $1 \mathrm{~mm}$ en la precipitación (Figura 5). 
Tecnología y

Ciencias $\asymp$ Agua
2020, Instituto Mexicano de Tecnología del Agua

Open Access bajo la licencia CCBY-NC-SA 4.0

(https://creativecommons.org/licenses/by-nc-sa/4.0/)

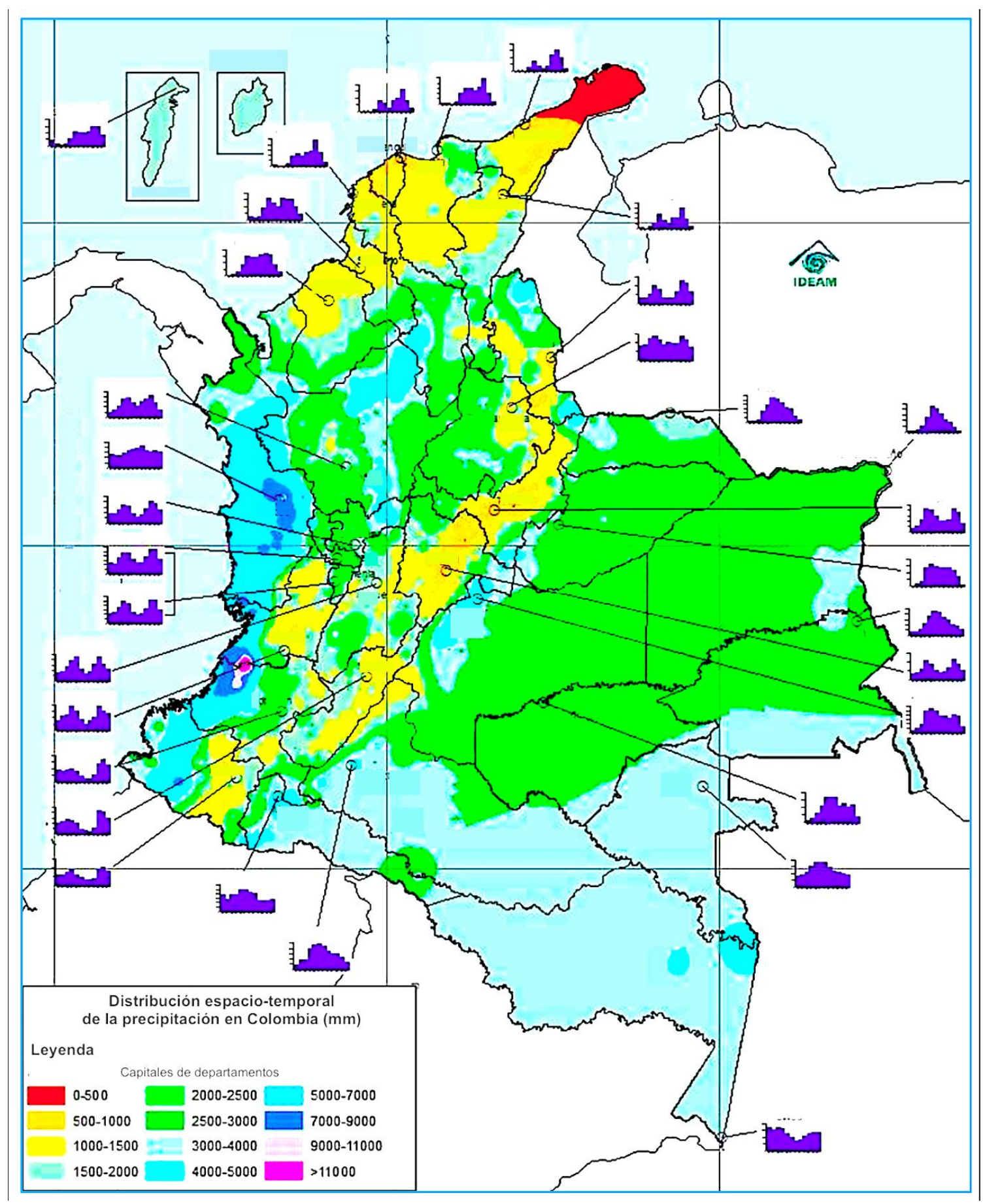

Figura 5. Mapa precipitación Colombia 1971-2000: Fuente: IDEAM (2011). 
Tecnología y

Ciencias $₫$ Agua
2020, Instituto Mexicano de Tecnología del Agua

Open Access bajo la licencia CC BY-NC-SA 4.0

(https://creativecommons.org/licenses/by-nc-sa/4.0/)

\section{Estructura tarifaria}

Alude a la estructura tarifaria cuando se estudian reglas y procedimientos que determinan el cobro de un servicio a diferentes categorías de consumidores (Brocklehurst, 2002). Algunos elementos de dicha estructura son tarifa fija, volumétrica $y$, en algunos casos, la tarifa multipartes. Dentro de la tarifa fija se considera el precio del agua que, si bien ha despertado interés por definir la influencia de esta variable sobre la demanda de agua potable, no entra en consideración para el proyecto, pues el usuario no llega a saber el valor real del $\mathrm{m}^{3}$ de agua, ya que el valor facturado es parte de un cálculo que contempla elementos como subsidios y costos administrativos, entre otros. Otra razón por la que se decidió no incluir el precio del agua está dada por la inelasticidad de esta variable sobre la demanda. El precio se estudia por medio de un modelo de metarregresión donde se demostró que no influye en la demanda (Dalhuisen, Florax, DeGroot, \& Nijkamp, 2003). Por esta razón es tan importante tener una estructura tarifaria razonable y adecuada con las necesidades de los distintos sectores del país, convirtiéndose en un determinante fundamental para el cálculo de la demanda de agua. Según los estudios de caso consultados para Colombia, se encontró que la Resolución 08 de 1995 de la Comisión de Regulación de Agua Potable y Saneamiento Básico (CRA) estableció una estructura de tarifas basada en 
bloques de consumo, para que las empresas proveedoras calculen los precios de cada bloque, denominada bloques crecientes de consumo $(B C C)$, que se relacionan a través de un sistema de subsidios cruzados. Esta estructura de tarifas considera tres bloques de consumo: el básico, en el rango de 0 a $20 \mathrm{~m}^{3} / \mathrm{mes} / \mathrm{suscriptor}$; el complementario, de 21 a 40 $\mathrm{m}^{3}$, y el suntuario, mayor a $40 \mathrm{~m}^{3} / \mathrm{mes} /$ suscriptor.

\section{Cambio climático}

Afecta la demanda de agua, al poder causar variaciones del clima de forma constante, modificando de diferentes maneras y magnitudes la demanda. La importancia de este determinante se debe a distintos pronósticos sobre variables, como precipitación y temperatura media superficial del planeta (IPCC, 2013), llegando a cambiar la frecuencia y afectando también la prevalencia de eventos de clima extremos, como sequías o periodos de lluvias de mayor intensidad; el ciclo del agua (Buytaert, Cuesta, \& Tobón, 2011), y la disponibilidad de agua para consumo humano (CEPAL, 2000).

De acuerdo con estudios consultados para Colombia, estos valores corresponden a predicciones realizadas con la ayuda de series de temperatura y precipitación para diferentes periodos, como el de 1997 a 2013, donde se proyectaron cuatro escenarios futuros, que al compararlos con periodos entre 2014 y 2030 (con corrida de modelos), los resultados 
identificaron una disminución leve en el consumo de agua, planteando relaciones por el aumento en el forzamiento radiactivo por mayores emisiones de gases efecto invernadero a la atmósfera. Esta reducción mensual en el consumo esperado de agua en los cuatro escenarios de cambio climático establecido no fue mayor de $2 \%$. En el método uno, la disminución del consumo fue de $0.33 \mathrm{~m}^{3}$; para el método dos, la baja fue de $0.26 \mathrm{~m}^{3}$. De igual manera, resalta que estos efectos potenciales del cambio climático sobre la demanda de agua para uso residencial urbano podrían ser importantes en áreas en las que el consumo discrecional sea significativo con respecto a la demanda, o también en sitios caracterizados por periodos secos prolongados y con crecientes demandas por aumento de la población. Gracias a tales investigaciones resulta necesario incluir este determinante en el cálculo de la demanda de agua, pues por su variabilidad cambia el consumo.

\section{Características de la vivienda}

Este determinante se relaciona con la composición interna y externa de la vivienda donde habita cada usuario y también con el número de personas que la habiten, ya que conforme estas condiciones, la demanda de agua puede variar dependiendo de las características. En el estudio aplicado para Manizales (Colombia), se demostró una dependencia del tipo de vivienda; si es casa o apartamento, el consumo es diferente; si es 
una casa, el consumo aumenta $34 \%$ más que el consumo en un apartamento. Una causa, según el estudio, es mayor demanda de agua para el uso discrecional, específicamente el relacionado con lavado de patios y riego de jardines. Otro posible motivo que aumenta la demanda es el número de baños, pues sube en un 3.8\%. Para el uso no discrecional (preparación de alimentos y aseo personal), en este estudio también resultó que la mayor cantidad de baños en una vivienda fue cuatro. Otras investigaciones consiguieron signos positivos en la variable de números de baños, como en EUA y Canadá (Olmstead, Hanemann, \& Stavins, 2007); México (Jaramillo, 2005), y Colombia (Medina \& Morales, 2007). Del mismo modo, aparatos como lavadoras incrementan la demanda de agua en $11 \%$.

\section{Variables no determinantes aplicables para Colombia}

Políticas. Por medio de esta variable se determinan las metodologías, estructuras y reglamentación relacionada con la demanda del agua, pues si no se sigue lo estipulado, no tiene ninguna validez o sustento ante la sociedad y se desprende de la gobernanza. Otros criterios aplicables no determinantes para Colombia son:

a) Accesorios como piscinas, jardines. Tienen ligera influencia en la demanda (Wentz \& Gober, 2007); se encontró que un aumento de la 
unidad en el porcentaje de casas con piscinas genera un incremento en el promedio del consumo anual de agua de hasta un $1 \%$ para la zona de Phoenix, Arizona, EUA. Para el caso de Colombia, no genera afectación significativa.

b) Evapotranspiración. Se entiende como la combinación de dos procesos separados, a través de los cuales el agua se pierde en la superficie del suelo por evaporación y transpiración del cultivo (Allen, Pereira, Raes, \& Smith, 2006). Asimismo, existe la evapotranspiración potencial, producida por la humedad del suelo y la cobertura vegetal en condiciones óptimas (Thornthwaite, 1948). Si bien este criterio afecta la demanda del agua (Howe \& Linaweaver, 1967; Agthe \& Billings, 1980, 1987; Maidment \& Parzen, 1984), en Colombia no representa una variación significativa, dado que el tipo de suelos en las regiones urbanas está estandarizado sobre el suelo construido.

c) Establecimiento de indicadores para determinantes. Por lo señalado, la estructura del sistema para la proyección de demanda se construye sobre cuatro determinantes: escenarios de cambio climático, estructura tarifaria, vivienda y clima (Tabla 2 ).

Tabla 2. Relación de indicador(es) por determinante.

\begin{tabular}{|c|c|}
\hline Determinante & Indicador \\
\hline $\begin{array}{c}\text { Escenarios de } \\
\text { cambio }\end{array}$ & Proyección favorable y desfavorable de variables \\
climático & climatológicas \\
\hline
\end{tabular}




\begin{tabular}{|c|c|}
\hline $\begin{array}{c}\text { Estructura } \\
\text { tarifaria }\end{array}$ & $\begin{array}{r}\text { Tarifa del servicio de acueducto (cargo por } \\
\text { consumo) }\end{array}$ \\
$\begin{array}{c}\text { Precio del agua (con factores de subsidios, } \\
\text { aportaciones solidarias e inversiones sociales). }\end{array}$ \\
\hline \multirow{2}{*}{ Vivienda } & $\begin{array}{r}\text { \#Personas/año/vivienda de déficit habitacional cabeceras } \\
\text { internas de la misma. }\end{array}$ \\
\cline { 2 - 3 } & Variación anual de variables temperatura y \\
Clima & precipitación \\
\hline
\end{tabular}

\section{Resumen metamodelo}

El meta modelo utilizado fue un sistema de soporte de decisiones (DSS) incorporando una red neuronal artificial (ANN), que contempla determinantes con sus respectivos indicadores: a) determinantes: cambio climático, vivienda, estructura tarifaria y clima; b) indicadores: escenarios de cambio climático, \% déficit de vivienda cualitativo, \% de hacinamiento no mitigable, tarifa $/ \mathrm{m}^{3}$, temperatura y precipitación. 


\section{Medición}

$$
\text { Cantidad de dispositivos }=\frac{\left(\frac{\text { Ahorradores }}{\text { No ahorradores }}\right)}{\text { Cantidad de dispositivos en el área urbana }}
$$

\# personas por vivienda, $\$$ tarifa $\mathrm{m}^{3}$-políticas que fomenten o no ahorro.

Fórmula de precipitación:

$$
\begin{aligned}
& L n W_{i t}=\beta_{0}+\beta_{1} * \ln (\text { MPit })+\beta_{2} * \ln (\text { Dit })+\beta_{3} * \ln (\text { INCit })+\sum_{i=4}^{u} \beta_{i}(N P D S M)+\beta_{3} * \\
& \ln (\text { PRECit })+L O T
\end{aligned}
$$

Donde:

$i=1, \ldots, 8$ ciudades.

$t=1, \ldots, 96$ meses (tiempo).

$W_{i t}=$ demanda residencial mensual .

$D_{i t}=$ diferencia variable (la diferencia entre lo que hubiera pagado si todos los lotes hubieran sido cotizados a precio del mercado y el precio pagado condiciones de bloque).

$I N C_{i t}=$ ingreso en múltiplos de $\$ 100,000$.

$\mathrm{Hh}_{\text {it }}=$ número de miembros del hogar . 
NPDSM = 8 (políticas de administración del tamaño de la demanda). PREC $=$ precipitación $\left(\mathrm{m}^{3} / \mathrm{mes}\right)$.

Fórmula de temperatura:

$$
d_{\tau}=\frac{\Delta L W}{\Delta T}
$$

Donde $L W$ es el cambio en la demanda de agua por la variación temperatura $(\Delta T)$, el cual indica que la manera más apropiada está relacionada con registros históricos en las áreas urbanas, como ya se había determinado; el impacto de variables climatológicas, y la influencia de manera moderada de la demanda de agua.

\section{Discusión}

La construcción del metamodelo se realizó haciendo énfasis en la necesidad de dar prioridad a fenómenos ambientales, pues su efecto va adquiriendo mayor relevancia en el comportamiento de los habitantes de áreas urbanas; la vulnerabilidad ante eventos climáticos extremos es cada vez más frecuente, y se genera incertidumbre frente a estimaciones de 
demanda de agua potable a largo plazo. Los municipios que sirvieron como base para el metamodelo fueron aquellos en los que existen datos históricos que pudieron alimentar las funciones matemáticas expuestas; de lo contrario, las proyecciones no se hubieran ajustado a la realidad de la zona a implementarse, y hubieran conducido a la toma de decisiones no favorables en lo administrativo, operativo y técnico para el área urbana.

El modelo se plantea para proyectar la demanda de agua potable en horizontes a largo plazo para zonas urbanas. Por tanto, la primera delimitación que se hizo durante la selección y el planteamiento del metamodelo se enfocó en el sector geográfico. Sin embargo, los modelos que alimentan el metamodelo requieren de las bases de datos de las empresas de servicios públicos, en especial de acueducto, pues a partir de su información se posibilita la existencia de indicadores que estructuran las redes neuronales artificiales. Es por ello que las figuras de acueductos veredales, comunitarios o municipales que no cuenten con indicadores operativos, administrativos o técnicos, no se incluirán en la aplicación de la simulación del metamodelo. Asimismo, el supuesto establece que a partir de datos históricos puede extrapolarse su comportamiento, pero no asegura que se cumpla, y por tanto el riesgo es alto.

El metamodelo debe simularse en varios softwares para garantizar que las proyecciones tengan intervalos de confianza de mínimo 95\%, que tenga una varianza mínima y que la correlación de los datos sea cercana a uno (1). Algunos softwares recomendados para la simulación son: 
1. Matlab ${ }^{\circledR}$.

2. Demand Works ${ }^{\circledR}$.

3. Oracle ${ }^{\circledR}$.

4. Onestreamsoftware ${ }^{\circledR}$.

5. $R^{\circledR}$.

Otro factor de restricción son las políticas, debido a que una eventual acogida no significa su implementación, por ello es indispensable que ésta cuente con articulación, así como con los entes gubernamentales y ejecutivos. Ello se respalda en el marco normativo que provee a las empresas de herramientas que incentiven prácticas de eficiencia y calidad.

\section{Conclusiones}

El metamodelo planteado suple una necesidad de brindar herramientas al sector público-privado para regular aspectos técnicos y normativos del servicio de acueducto a partir de una proyección adecuada y, sobre todo, vinculante con aspectos medio-ambientales, que en zonas urbanas se convierten en un aspecto crítico con respecto a su planeación y su administración; sin embargo, el éxito del planteamiento depende de la aplicación apropiada y ajustada a la situación de cada ciudad o municipio. 
Para la investigación de datos y teorías acerca de metamodelos y la proyección de demanda de agua en el contexto internacional se encontró poca información, a pesar de ser un tema tratado desde hace más de tres décadas. La información sobre la oferta de agua potable, útili para el complemento del estudio, sí cuenta con registros en bases de datos. En el ámbito nacional tampoco hay documentación robusta ni investigación acerca de los metamodelos aplicados a una región específica. Del mismo modo, en el contexto internacional se encontró literatura acerca de la proyección de la demanda de agua, principalmente de la región andina. Las determinantes elegidas surgieron de un análisis de las diferentes afectaciones y condiciones del territorio colombiano en sus áreas urbanas y de la revisión de la literatura encontrada acerca del proyecto, con el fin de adoptarlas al metamodelo, para así ajustarlas y con ello obtener un resultado confiable en la proyección de la demanda de agua y próximo al real, incluyendo determinantes que generan afectación del recurso hídrico en el horizonte a largo plazo.

Para integrar las determinantes en el metamodelo se establecieron sus respectivos indicadores, con la función principal de tener claridad en la dimensión y afectación de cada una de ellas en el horizonte de proyección a largo plazo de la demanda de agua, aportando mayor peso a las determinantes de cambio climático y meteorológicas. Estas dos cumplen con un papel fundamental en los cambios que puede tener la demanda por sus variaciones, como es el caso del cambio climático, por esto es que comprenden mayor robustez en su sustento. Es importante tener en cuenta dicha afectación, pues tales determinantes pueden tener cambios en el tiempo y su comportamiento no es extrapolable bajo condiciones de linealidad. Otra determinante que influyó en los cálculos 
de la proyección de la demanda fue el rango de consumos, donde se realizaron simulaciones para dotaciones de agua para satisfacer el mínimo vital ( $5 \mathrm{~m}^{3}$ para viviendas de estrato bajo-bajo y $3 \mathrm{~m}^{3}$ para estrato bajo) para poblaciones de estratos bajos, con tarifas cercanas a cero pesos; con un rango básico subsidiado (para estratos bajos) de hasta $20 \mathrm{~m}^{3}$ al mes, con particiones por clima (por debajo de 1000 m de altitud, entre 1000 a 2 000, y más de 2 000, con diferenciales entre 2 y $3 \mathrm{~m}^{3}$ por piso térmico); con un rango complementario de dos veces cada franja de volumen en rango básico, y un rango elevado denominado suntuario, con costos incrementales de largo plazo.

La delimitación para el metamodelo se sienta en su aplicación, que sólo fue diseñada para entornos urbanos y con planes a largo plazo. Se tuvieron en cuenta restricciones, como la figura de acueductos veredales comunitarios o municipales, que en su mayoría no tiene indicadores operativos, administrativos o técnicos, y por lo tanto no se pueden usar en la simulación del metamodelo, donde también hay un error e incertidumbres asociadas para poder estimar su exactitud y precisión, para que así el valor de la demanda de agua sea el más real para el territorio en estudio. De la ampliación de la investigación científica mencionada se plantean los siguientes estudios científicos: metamodelo de regulación para calcular índices de agua no contabilizada y metamodelo de regulación para precios techo en servicios públicos de saneamiento básico.

\section{Agradecimientos}


Los autores agradecen a la Universidad Distrital Francisco José de Caldas de Bogotá, Colombia, donde inició esta investigación, y a la Universidad de Guadalajara de México, donde se concluyó.

\section{Referencias}

Adamowski, J. (2008). Peak daily water demand forecast modeling using artificial neural networks. Journal of Water Resources Planning and Management, 134(2). DOI: 10.1061/(ASCE)07339496(2008)134:2(19).

Agthe, D. E. \& Billings, R. B. (1980). Dynamic models of residential water demand. Water Resources Research, 16(3), 476-480. Recuperado de https://doi.org/10.1029/WR016i003p00476

Agthe, D. E. \& Billings, R. B. (1987). Equity, price elasticity, and household income under increasing block rates for water. American Journal of Economics and Sociology, 46(3), 273-286. Recuperado de https://www.jstor.org/stable/3486079

Allen, R. G., Pereira, L. S., Raes, D., \& Smith, M. (2006). Evapotranspiración del cultivo. Guías para la determinación de los requerimientos de agua de los cultivos. Estudio Riego y Drenaje, FAO-56. Recuperado de fao.org/3/x0490s/x0490s.pdf

Balling, R. C., \& Gober, P. (2007). Climate variability and residential water use in the city of Phoenix, Arizona. Journal of Applied Meteorology and Climatology, 46, 1130-1137. Recuperado de https://doi.org/10.1175/JAM2518.1 
Bougadis, J., Adamowski, K., \& Diduch, R. (2005). Short-term municipal water demand forecasting. Hydrology Processes, 19, 137-148. DOI:10.1002/hyp.5763.

Brocklehurst, C. (2002). New designs for water and sanitation transactions: Making private sector participation work for the poor. Washington, DC, USA: Public-Private Infrastructure Advisory Facility.

Buytaert, W., Cuesta, F. \& Tobón, C. (2011). Potential impacts of climate change on the environmental services of humid tropical alpine regions. Global Ecology and Biogeography, 20 (1), 19-33. Recuperado de https://doi.org/10.1111/j.1466-8238.2010.00585.x

Caiado, J. (2010). Performance of combined double seasonal univariate time series models for forecasting water demand. Journal of Hydrologic Engineering, 15(3). DOI: 10.1061/(ASCE)HE.19435584.0000182

CEPAL, Comisión Económica para América Latina y el Caribe. (2000). Agua para el siglo XXI para América del sur. De la visión a la acción. Recuperado de https://www.cepal.org/samtac/noticias/documentosdetrabajo/5/2 3345/InCo00200.pdf

Cutore, P., Campisano, A., Kapelan, Z. \& Savic, D. (2007). Stochastic forecasting of urban water consumption using neural networks and the SCEM-UA algorithm. In: Ulanicki, B., Vairavamoorthy, K., Butler, D., Bounds, P. L. M., \& Ali-Memon, F. (eds.). Water management challenges in global change. pp. 371-377). Leicester, UK: Taylor \& Francis Group. 
Dalhuisen, J., Florax, R. J. G. M., DeGroot, H. L. F., \& Nijkamp, P. (2003). Price and income elasticities of residential water demand: A metaanalysis. Land Economics, 79(2):292-308. DOI: 10.2307/3146872.

Gilpin, A. (2000). Environmental economics: Acritical overview. New York, USA: John Wiley \& Sons.

Hamilton, J. D. (1994). Times series analysis. New Jersey, USA: Princeton University Press.

Howe, C. W., \& Linaweaver, F. P. (1967). The impact of price on residential water demand and its relation to systems design and Price structure. Water Resources Research, 3(1), 13-32. Recuperado de https://doi.org/10.1029/WR003i001p00013

IDEAM, Instituto de Hidrología, Meteorología y Estudios Ambientales. (2010). Índice de Alteración Potencial de la Calidad de Agua en condiciones hidrológicas de año medio. Sub-zonas hidrográficas. Bogotá, Colombia: Instituto de Hidrología, Meteorología y Estudios Ambientales.

IDEAM, Instituto de Hidrología, Meteorología y Estudios Ambientales. (2011). Mapas de precipitación promedio en colombia. Recuperado de http://www.ideam.gov.co/documents/21021/21141/precip+media +\%5BModo+de+compatibilidad\%5D.pdf/e0ae03be-8e3a-44f8b5a2-2148a5aeff4d

IDEAM, Instituto de Hidrología, Meteorología y Estudios Ambientales. (2015). Estudio Nacional de agua 2014. Bogotá, Colombia: Instituto de Hidrología, Meteorología y Estudios Ambientales. 
IPCC, Intergovernmental Panel on Climate Change. (2013). Climate change 2013: The physical science basis. Contribution of working Group I to the Fifth Assessment Report of the Intergovernmental Panel on Climate Change. Cambridge, UK: University Press. Recovered from https://www.ipcc.ch/site/assets/uploads/2018/03/WG1AR5_Sum maryVolume_FINAL.pdf

Jaramillo, L. (2005). Evaluación econométrica de la demanda de agua de USO residencial en México. El Trimestre Económico, 72(2), 367390. DOI: $10.2307 / 20856859$

Kuo, R. J., \& Xue, K. C. (1998). A decision support system for sales forecasting through fuzzy neural networks with asymmetric fuzzy weights. Decision Support System, 24(2), 105-126. Recuperado de https://doi.org/10.1016/ S0167-9236(98)00067-0

Lenard, M., Alam, P., \& Madey, G. (1995). The applications of neural networks and a qualitative response model to the auditors going concern uncertainty decision. Decision Sciences, 26(2), 209-227. Recuperado de https://doi.org/10.1111/j.15405915.1995.tb01426.x

Lizcano, J. A. (2011). Investigación al régimen tarifario de agua potable y saneamiento básico. Bogotá, Colombia: Universidad Distrital Francisco José de Caldas.

Maidment, D. R., \& Parzen, E. (1984). Time patterns of water use in six Texas cities. Journal of Water Resources Planning and Management, 110(1). DOI: 10.1061/(ASCE)0733-9496(1984)110:1(90) 
Medina, C., \& Morales, L. F. (2007). Demanda por servicios públicos domiciliarios en Colombia y subsidios: implicaciones sobre el bienestar. Borradores de Economía, (467) Recuperado de https://www.banrep.gov.co/es/demanda-servicios-publicosdomiciliarios-colombia-y-subsidios-implicaciones-sobre-elbienestar

Michie, D., Spiegelhalter, D. J., \& Taylor, C. C. (1994). Machine learning, neural and statistical classification. Recuperado de https://www1.maths.leeds.ac.uk/ charles/statlog/whole.pdf

Ministerio de Ambiente, Vivienda y Desarrollo Territorial. (2003). Definición del nivel de complejidad y evaluación de la población, la dotación y la demanda de agua. Guía RAs 001 2000. Recuperado de http://www.minvivienda.gov.co/GuiasRAS/RAS\%20-\%20002.pdf

Nijkamp, P. (1977). Theory and application of environmental economics. Amsterdam, The Netherlands: North Holland Publishing Company.

Olmstead, S. M., Hanemann, W. M., \& Stavins, R. N. (2007). Water demand under alternative price structures. Journal of Environmental Economics and Management, 54(2), 181-198, Recuperado de https://doi.org/10.1016/j.jeem.2007.03.002

OMS, Organización Mundial de la Salud. (2015). Organización Mundial de la Salud. Recuperado de http://www.who.int/water_sanitation_health/monitoring/jmp2015-key-facts/es/

Patuwo, E., Hu, M. Y., \& Hung, M. S. (1993). Two-group classification using neural networks. Decision Sciences, 24(4), 825-845. 
Tecnología y

Ciencias $₫$ Agua
2020, Instituto Mexicano de Tecnología del Agua

Open Access bajo la licencia CCBY-NC-SA 4.0

(https://creativecommons.org/licenses/by-nc-sa/4.0/)

Recuperado de https://doi.org/10.1111/j.15405915.1993.tb00491.x

Pearce, D. W., \& Turner, R. K. (1990). Economics of natural resources and the environment. Baltimore, USA: The Johns Hopkins University Press.

Thornthwaite, C. W. (1948). An approach toward a rational classification climate. Geographical Review, 38(1), 55-94. DOI: 10.2307/210739

Wentz, E., \& Gober, P. (2007). Determinants of small-area water for the city of Phoenix, Arizona. Water Resources Management, 21, 18491863. Recuperado de https://doi.org/10.1007/s11269-006-9133-0 